\title{
Solution Viscosity Behavior and Swelling Behavior of Polystyrene-Based Cationic Ionomers. Effects of Added Salts and Counterion
}

\author{
Noritaka Ohtani, * Yukihiko Inoue, Yasumasa Kaneko, Akiko SaKakida, \\ Ichiro TAKeISHI, and Hiroshi Furutani
}

Department of Materials Engineering \& Applied Chemistry, Akita University, Akita 010, Japan

(Received June 21, 1995)

\begin{abstract}
Dilute-solution viscosity was investigated for several polystyrene-based cationic ionomers in the presence of small salts. The results were compared with the swellability of the corresponding crosslinked ionomers. It was found that intra- and intermolecular aggregation among the ionic groups in nonpolar solvents was eliminated by the addition of the low-molecular quaternary salts, leading to increases in the solubility and the reduced viscosity of the linear ionomers as well as an increased swelling of the crosslinked ionomers. Polyelectrolyte behavior in polar organic solvents was suppressed by the minute addition of soluble salts, resulting in a decreased reduced viscosity of linear ionomers and a decreased swelling of crosslinked ionomers. These results are discussed in terms of the change in the intra- and intermolecular aggregation of quaternary salts of the ionomers.
\end{abstract}

KEY WORDS Cationic Ionomers / Polystyrene-Based Ionomers / Solution Viscosity / Quaternary Salts Solvation / Aggregation of Ionic Groups /

Ionomers are a class of linear polymers containing a relatively low level of ionic groups. The solution viscosity behavior of anionic ionomers has been extensively studied because of their importance as industrial materials. However less information is available about cationic ionomers, in spite of the excellent usefulness of the corresponding crosslinked ionomers as efficient phase-transfer catalysts. ${ }^{1-10}$

We have clarified the dilute solution behavior of polystyrene-based cationic ionomers containing ammonio or phosphonio groups ${ }^{11}$ and have related it with their reactivities for nucleophilic substitution reactions. ${ }^{10}$ Polyelectrolyte behavior was generally observed in polar solvents with high dielectric constants. This is a clear evidence for the presence of dissociated ions in the ionomer solution, while various approaches have been taken to the interpretation of polyelectrolyte effect..$^{12-14}$ On the other hand, the formation of intramolecular aggregates among the ionic groups takes place in nonpolar solvents, decreasing the reduced viscosity of the ionomer solution, which leads to the reduction of the apparent reactivity of the quaternary ammonium or phosphonium chloride. The larger the ion content, the greater the extent of the ionic aggregation. The intramolecular aggregation is dependent on the solvation to the quaternary cations and to the counter-anions. ${ }^{15}$ The solvents with high values of acceptor numbers, $A_{\mathrm{N}},{ }^{16}$ strongly solvate the counterions, reducing the apparent nucleophilic reactivity, while the aggregation of ionic groups is inhibited in these solvents due to the strong solvation. The solvents with high values of donor number, $D_{\mathrm{N}}{ }^{16}$ solvate the quaternary cations, also leading to a lowering aggregation. When an ionomer consists of small quaternary cations or small counter-anions, therefore, an extensive aggregation occurs if the solvent has little affinities either to cations or to anions.

Although aggregation behavior has been evidenced for ionomer solutions in nonpolar solvents, the behavior in relatively polar solvents has not been understood. The size of counterions and the presence of small salts may strongly influence the ionic aggregation even in polar solvents. There are some quaternary salts that are soluble in nonpolar solvents. Therefore, it is very interesting to investigate the influence of such salts on the ionic aggregation in nonpolar solvents.

In this study, it is shown that the solubility and the dilute solution viscosity of polystyrene-based cationic ionomers with ammonio or phophonio groups are varied by the addition of low-molecular-weight salts. We particularly describe the interaction of the ionomerattached quaternary groups with soluble low-molecular quaternary salts. We discuss the change in the intra- and intermolecular aggregations of linear and crosslinked ionomers by the addition of the quaternary salts.

\section{EXPERIMENTAL}

\section{Materials and General Methods}

$N, N$-Dimethylbutylamine and $N, N$-dimethyloctylamine were prepared by the $N$-methylation of butylamine and octylamine, respectively, by means of formic acid and formalin, and were purified by distillation under nitrogen. Styrene and vinylbenzyl chloride (60/ 40 , meta/para) were distilled under reduced pressure prior to use. Tributylhexadecylphosphonium chloride (TBHPC) and tributylhexadecylphosphonium bromide (TBHPB) were synthesized by the reactions of tributylphosphine with hexadecylchloride and hexadecylbromide, respectively, and were purified by recrystallization from diethylether solutions. Tributylhexadecylphosphonium iodide (TBHPI) was prepared by the reaction of TBHPB with methyl iodide. Benzyltributylphosphonium chloride (BTBPC) was prepared by the reaction of tributylphosphine with benzylchloride in toluene at $90^{\circ} \mathrm{C}$ and was recrystallized from toluene. Cetyltrimethylammonium chloride (CTAC), benzylhexadecyldimethylammonium chloride (BHDAC), and methyltrioctylammonium chloride (TOMAC) were commercially obtained from Tokyo Kasei Ltd. Potassium thiocyanate was recrystallized from ethanol. Other 
inorganic salts were all guaranteed grade and were used without further purification. Toluene and tetrahydrofuran (THF) were purified by distillation from sodium benzophenone ketyl under nitrogen. Methanol was dried over magnesium activated with iodine. Other organic solvents were dried and distilled in a usual manner.

Dye solubilization measurement was done using sudan III, 1-[[4-(phenylazo)phenyl]azo]-2-naphthalenol, at $25^{\circ} \mathrm{C}$ on HITACHI U-2000A spectrophotometer.

\section{Polymer Preparation}

Linear copolymers of styrene and vinylbenzyl chloride (LCM $x$ PS) were prepared by the method reported previously and their average molecular weights were estimated from their intrinsic viscosities in toluene at $25^{\circ} \mathrm{C}$ using the viscosity-molecular weight relationship given for homopolystyrene; the equation, $[\eta]=(1.7 \times$ $\left.10^{-4} \mathrm{dL} \mathrm{g}^{-1}\right) M^{0.69} \cdot{ }^{17}$ These linear copolymers were reacted with a five fold excess of an $N, N$-dimethylalkylamine or tributylphosphine in toluene at $60^{\circ} \mathrm{C}$. The resulting linear ionomers, LA $x \mathrm{RCl}$ and $\mathrm{LP} x \mathrm{BuCl}$, were purified by two reprecipitations from dichloromethane into petroleum ether and dried. The content of ammonium chloride or phosphonium chloride was determined by GLC by analyzing the amount of 1-chlorodecane that was formed through the reaction of the ionomers with excess decyl methanesulfonate in toluene at $90^{\circ} \mathrm{C}$. In Table I, the typical ionomers used in this study are listed. The ionomers with iodide counter ions were prepared by the reaction of an ionomer, $\mathrm{LA} 9 \mathrm{OcCl}$, with excess methyl iodide in benzene at $60^{\circ} \mathrm{C}$ for $24 \mathrm{~h}$. The obtained ionomer was purified by reprecipitation from dichloromethane into petroleum ether. The ionomers with mesylate and tosylate counter ions were prepared by the reaction of the chloride ionomers with excess methyl mesylate and methyl tosylate, respectively. The ionomers with other counter ions were prepared by the ion-exchange method that the methanol solution of the chloride ionomer was equilibrated with the corresponding solid sodium salts.

In a similar manner, the crosslinked ionomers,

Table I. The typical ionomers and the minimum concentration of quaternary salts $(\mathrm{QX})$ dissolving the ionomers in toluene or tetrahydrofran

\begin{tabular}{|c|c|c|c|}
\hline \multirow{2}{*}{ Ionomer $(\overline{D P})^{\mathrm{a}}$} & \multirow{2}{*}{ QX } & \multicolumn{2}{|c|}{$\begin{array}{l}\text { Mininum Concn of QX } \\
\qquad / \mathrm{mol} \mathrm{dm}^{-3}\end{array}$} \\
\hline & & Toluene & Tetrahydrofran \\
\hline LA7.5OcCl (350) & None & 0 & 0 \\
\hline \multirow[t]{2}{*}{$\mathrm{LA} 10 \mathrm{OcCl}(350)$} & BHDAC & - & 0.003 \\
\hline & TOMAC & 0.059 & 0.030 \\
\hline \multirow[t]{2}{*}{$\mathrm{LA} 15 \mathrm{OcCl}(350)$} & BHDAC & 0.045 & 0.030 \\
\hline & TOMAC & 0.076 & 0.039 \\
\hline LP5.0BuCl (200) & None & 0 & 0 \\
\hline LP9.0BuCl (200) & ТВHPC & 0.004 & $<0.001$ \\
\hline LP14BuCl (200) & ТВНPC & 0.083 & 0.017 \\
\hline \multirow[t]{2}{*}{ LP29BuCl (200) } & ТВНPC & $>0.25$ & 0.080 \\
\hline & TOMAC & - & 0.13 \\
\hline
\end{tabular}

\footnotetext{
${ }^{a}$ The abreviation of ionomers, $\mathrm{LA} x \mathrm{RCl}$ or $\mathrm{LP} x \mathrm{BuCl}$; $\mathrm{L}$ represents linear, A ammonio, $\mathrm{P}$ phosphonio, $x$ ion content as percent substitution of benzene ring, $\mathrm{R}$ alkyl group of $N, N$-dimethylalkylamine, Bu butyl group of tributylphosphine, and $\mathrm{Cl}$ counter chloride ion, respectively. $\overline{D P}$ represents the degree of polymerization.
}

$\mathrm{A} 12 \mathrm{BuCl}$ and $\mathrm{P} 12 \mathrm{BuCl}$, were prepared by the quaternization of a crosslinked chloromethylated polystyrene resin (Bio-Rad SX-1, 1\% DVB, $-200+400$ mesh, chloride content $1.26 \mathrm{mmol} \mathrm{g}^{-1}$ ) with $N, N$-dimethylbutylamine or tributylphosphine. The counterions were exchanged by the reaction method as described above.

\section{Solution Viscosity and Swelling Measurements}

Solubilities in several solvents were examined at $25^{\circ} \mathrm{C}$ at the concentration level of $0.4 \mathrm{~g} \mathrm{dL}^{-1}$. The mixtures were vigorously stirred using a vortex mixer and were allowed to stand overnight if not otherwise stated.

Solution viscosity was measured on Shibayama AutoViscometer SS-290S using a Ubbelohde capillary viscometer placed in a temperature-controlled water bath at $25^{\circ} \mathrm{C}$. Reduced viscosity $\eta_{\text {red }}$ was calculated from $\left(t-t_{0}\right) / t_{0} c$, where $c\left(\mathrm{~g} \mathrm{dL}^{-1}\right)$ is the ionomer concentration and $t$ is the flow time through the capillary at the concentration of $c$. The ionomer solution was diluted successively by the solvent containing a given concentration of a low-molecular quaternary salt after a reproducible flow time was obtained.

Swelling of the crosslinked ionomers was observed using a $10 \mathrm{ml}$ graduated sediment tube with ground glass joint. After $0.25 \mathrm{~g}$ of a crosslinked ionomer and $5.0 \mathrm{ml}$ of a solution containing a given concentration of a low-molecular quaternary salt were equilibrated in the tube under mild stirring with an enamel-coated copper wire, the tube was left for $3 \mathrm{~h}$ at $25^{\circ} \mathrm{C}$ and the final sedimentation volume was read. The supernatant solution was diluted or concentrated successively by the addition of the solvent or the concentrated salt solution after a reproducible sedimentation volume was obtained.

\section{RESULTS AND DISCUSSION}

The solubility of a cationic ionomer is primarily determined by the extent of solvent solvation to the quaternary cations and the counter-anions of the ionomer. ${ }^{15}$ The solvation is strongly enhanced when the solvent is with a high donor number $D_{\mathrm{N}}$ and the size of the cation is small or when the solvent is with a high acceptor number $A_{\mathrm{N}}$ and the size of the counter-anion is small. Dielectric constant $\varepsilon_{\mathrm{r}}$ is not a principal factor determining the solubility of an ionomer but it becomes important when the ion content of the ionomer is high. The coincidence of solubility parameter $\delta$ between solvent and polystyrene is also important when the ion content is low.

Since methanol has a large $A_{\mathrm{N}}$ value and a high $\varepsilon_{\mathrm{r}}$ value, the solvent is a good solvent for the ionomers with hard anions with small sizes like chloride ions. ${ }^{11}$ In fact, $\mathrm{LA} 9 \mathrm{OcCl}$ and $\mathrm{LA} 9 \mathrm{OcBr}$ were soluble in methanol. However, it was found that the ionomers LA9OcX with large counter ions, $X$, such as iodide, thiocyanate, or tosylate ions were insoluble in methanol probably due to the poor affinity of methanol to the counterions and polystyrene backbone. The potassium salts of these anions showed a strong salting-out effect for the methanolic solution of $\mathrm{LA} 9 \mathrm{OcCl}$. Picric acid also induced the precipitation of $\mathrm{LA} 9 \mathrm{OcCl}$ from the methanol solution. The precipitation took place when the amounts of the 


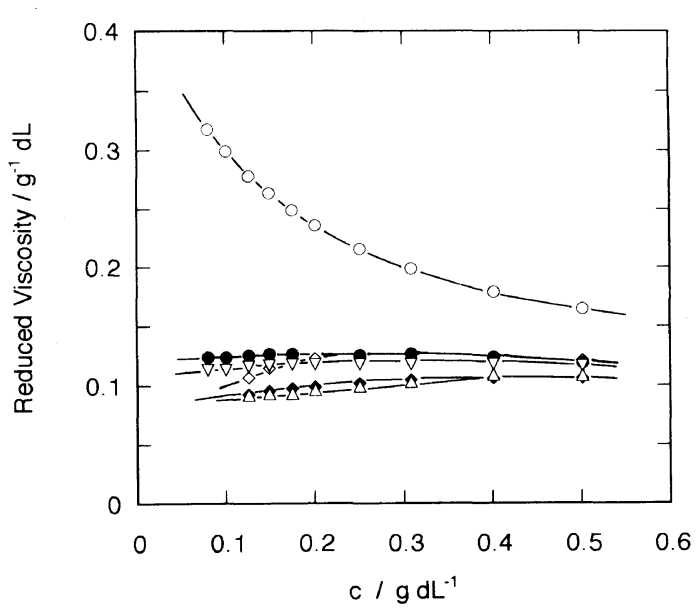

Figure 1. Solution viscosity behavior of $\mathrm{LP} 9 \mathrm{BuCl}$ in methanol with or without $1 \mathrm{mmol} \mathrm{dm}^{-3}$ of small salts at $25^{\circ} \mathrm{C}$ : (O) without salt, ( TBHPC, $(\diamond) \mathrm{BTBPC},(\nabla) \mathrm{KCl},(\diamond)$ Picric acid, and $(\triangle) \mathrm{KSCN}$.

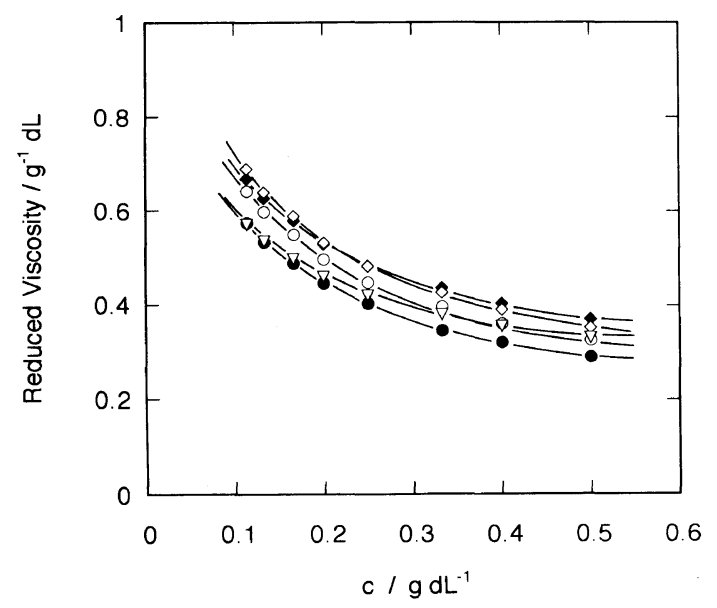

Figure 2. Solution viscosity behavior of LA7.5OcX in acetonitrile at $25^{\circ} \mathrm{C}: \mathrm{X}=\mathrm{Cl}(\bigcirc), \mathrm{Br}(\diamond), \mathrm{I}(\diamond), \operatorname{Tos}(\odot)$, and Mes $(\nabla)$.

added salts were almost equivalent to that of the ionomer-bound quaternary groups. Thus, the solution viscosity measurement was done at the salt concentration of $1.0 \mathrm{mmol} \mathrm{dm}^{-3}$, under which $\mathrm{LA} 9 \mathrm{OcCl}$ did not precipitate. As shown in Figure 1, the addition of a salt suppressed the polyelectrolyte behavior that was observed for salt-free solution. Similar elimination of polyelectrolyte behavior has been reported both for anionic ionomers in dimethylformamide ${ }^{12}$ and for cationic ionomers in acetonitrile. ${ }^{11}$ However, it is noted that the reduced viscosity was dependent on the type of added salt. This suggests that there is a certain specific interaction between the ionomer and the added salts.

The solubility of LA9OcX in acetonitrile was a little higher than in methanol. The solution viscosities of LA9OcX (X= Cl, Br, I, Tos, and Mes) in the absence of salts are shown in Figure 2. Each ionomer showed polyelectrolyte behavior in acetonitrile but the reduced viscosity was different from each other. This also suggests the specific interaction between the ammonio cation and the counter-anion.

A large size of counter-anions tends to decrease the extent of solvation, which may lead to an increasing formation of (contact) ion pairs if the solvation to the onium cation is also limited. The interaction of an ion

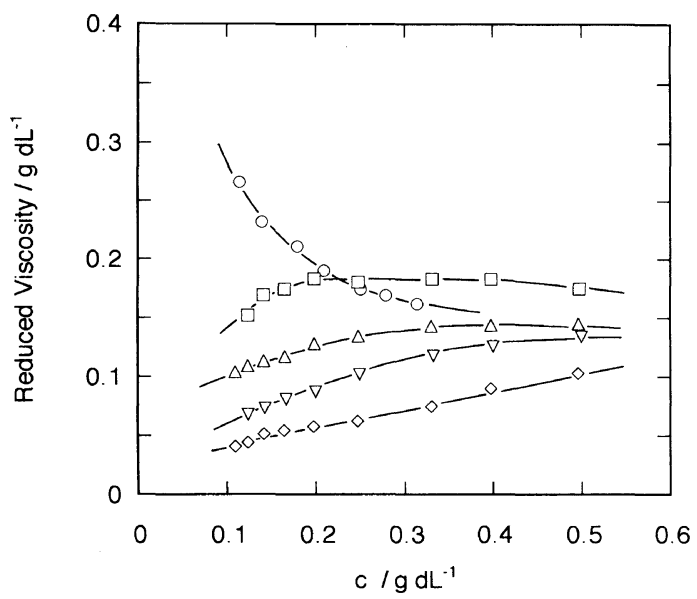

Figure 3. Solution viscosity behavior of $\mathrm{LA} 7 \mathrm{OcCl}(\overrightarrow{D P}=350)$ in water with and without CTAC at $25^{\circ} \mathrm{C}$ : concentration of CTAC, $0(\bigcirc), 0.5$ $(\square), 1.0(\triangle), 2.0(\nabla)$, and $3.0 \mathrm{mmol} \mathrm{dm}^{-3}(\diamond)$.

pair (a dipole) with an ammonio ion (a positive charge) is attractive. The interaction of an ion pair with another ion pair (another dipole) is also attractive. Thus, the intramolecular ionic aggregation becomes feasible, which results in the contraction of an ionomer chain. In methanol, its poor affinity to polystyrene-backbone may assist the contraction of a chain.

As reported previously, $\mathrm{LA} x \mathrm{OcCl}$ and $\mathrm{LP} x \mathrm{BuCl}$ with $x$ higher than $10 \%$ are easily dissolved in water if one replaces the solvent from methanol to water. ${ }^{11}$ This method was applied to examine the solubility of the ionomers with smaller ion contents. It was found that $\mathrm{LA} 5 \mathrm{OcCl}, \mathrm{LA} 7 \mathrm{OcCl}$, and $\mathrm{LP} 5 \mathrm{BuCl}$ were soluble in water by this method. We could make an aqueous solution even for LP5BuI, if the solvent was replaced from acetone (instead of methanol) to water. These ionomers showed polyelectrolyte-like behavior in the relation between the viscosity and concentration. As shown in Figure 3, the polyelectrolyte behavior of $\mathrm{LA} 7 \mathrm{OcCl}$ was suppressed by the addition of cetyltrimethylammonium chloride, the effect of which was similar to those of BTBPC and TBHPC observed for methanol solutions (Figure 1). However, the reduced viscosities were not always lower than that of the corresponding salt-free solution. The former was comparable to or slightly higher than the latter, when CATC concentration was lower than the CMC $\left(0.9 \mathrm{mmol} \mathrm{dm}^{-3}\right)$ and ionomer concentration was relatively high. Interestingly, the solubilization of sudan-III (an oil-soluble dye) into water was increased by the presence of $\mathrm{LA} 7 \mathrm{OcCl}$ in the whole range of CTAC concentrations as shown in Figure 4. These results indicate the importance of hydrophobic interaction between the ionomer chain and the surfactant molecules in addition to electrostatic interaction and also suggest the formation of polysoap-like structure in the solution. Surfactant cations may bind to polystyrene backbone in spite of the electrostatic repulsive force with ionomer ammonio cations and form a more hydrophobic domain around the ionomer chain.

Ionic aggregates are extensively formed in solvents with small $A_{\mathrm{N}}$ and low $\varepsilon_{\mathrm{r}}$ values, such as toluene and THF. ${ }^{11}$ The ionomers with a high ion content, e.g., $\mathrm{LA} 15 \mathrm{OcCl}$ and $\mathrm{LP} 14 \mathrm{BuCl}$, were insoluble and only swellable in these solvents probably due to an extensive 


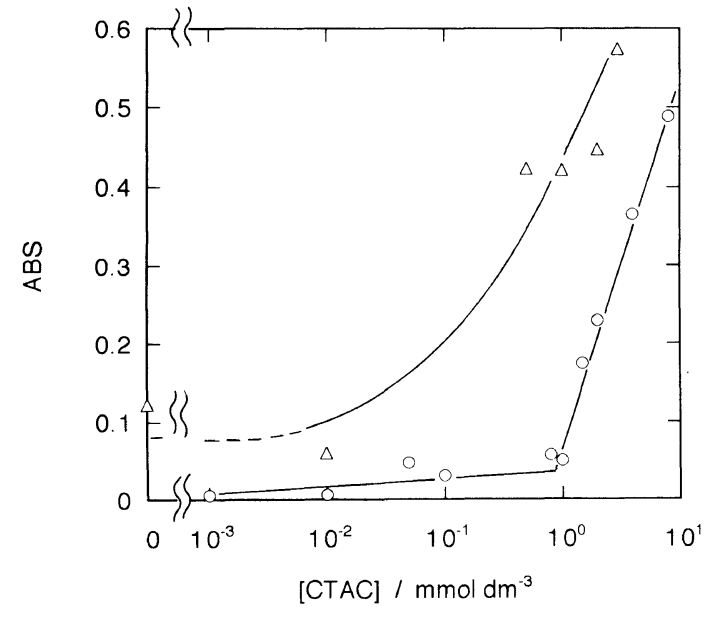

Figure 4. Solubilization of sudan III in water at $25^{\circ} \mathrm{C}$ : in the absence of $\mathrm{LA} 7 \mathrm{OcCl}(\bigcirc)$, and in the presence of $0.1 \mathrm{~g} \mathrm{dL}^{-1}$ of $\mathrm{LA} 7 \mathrm{OcCl}(\triangle)$.

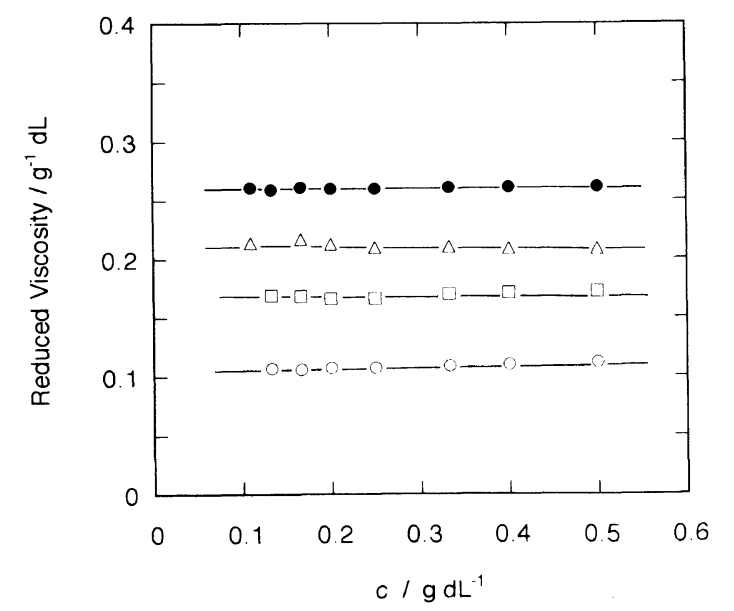

Figure 5. Solution viscosity behavior of LCM7.5PS and $\mathrm{LA} 7.5 \mathrm{OcCl}$ in THF at $25^{\circ} \mathrm{C}$ : (O) LCM7.5PS, (O) LA7.5OcCl, ( $\left.\square\right) \mathrm{LA} 7.5 \mathrm{OcCl}$ with $10 \mathrm{mmol} \mathrm{dm}^{-3}$ of TOMAC, and $(\triangle) \mathrm{LA} 7.5 \mathrm{OcCl}$ with $20 \mathrm{mmol} \mathrm{dm}^{-3}$ of TOMAC.

intermolecular aggregation. Interestingly, however, by adding low molecular-weight onium salts, such ionomers were solubilized in these nonpolar solvents. Salting-in phenomena of polymers are well-known, but the effect has been noted only for polar solvents using inorganic salts. As shown in Table I, the minimum concentration of an onium salt to dissolve an ionomer was generally smaller in THF than in toluene. The solubilizing power was dependent on the onium ion structure. BHDAC was more efficient for the salting-in effect, but the saturated concentration was necessary to solubilize $\mathrm{LA} 15 \mathrm{OcCl}$ in toluene. It is noted, however, that the toluene solution of $\mathrm{LA} 15 \mathrm{OcCl}$ solubilized by BHDAC was able to solubilize further amount of BHDAC above its solubility limit. The number of carbon atom of TOMAC was same as that of BHDAC but the ability was relatively poor, while a higher solubility of TOMAC in nonpolar solvents made it possible to dissolve the ionomers with large ion contents. Similarly, TBHPC readily solubilized phosphonio ionomers. It was impossible to evaluate the effect of BTBPC because of its low solubility in nonpolar solvents.

Figure 5 shows the effect of the TOMAC addition on the viscosity behavior of $\mathrm{LA} 7.5 \mathrm{OcCl}$ in THF. The reduced viscosities were nearly constant over the iono-

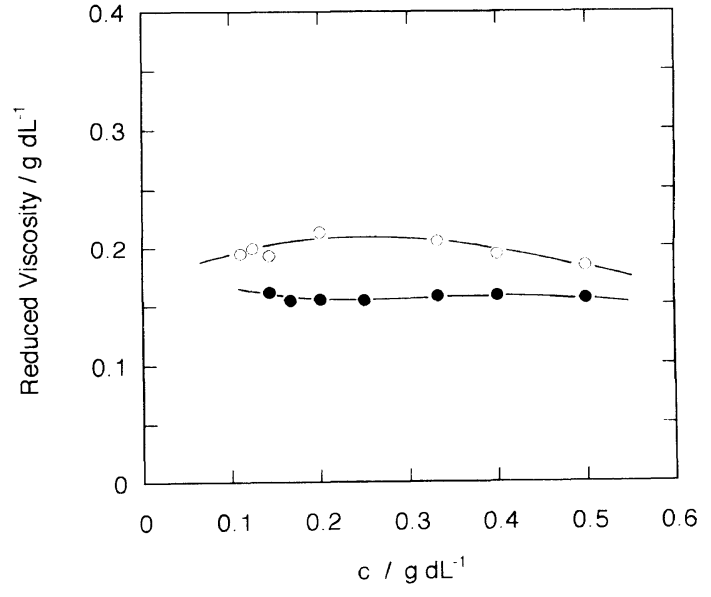

Figure 6. Solution viscosity behavior of $\mathrm{LP} 9 \mathrm{BuCl}$ in $\mathrm{THF}$ with TBHPC at $25^{\circ} \mathrm{C}$ with the TBHPC concentration at $20 \mathrm{mmol} \mathrm{dm}^{-3}(\bigcirc)$, and $50 \mathrm{mmoldm}^{-3}(\bigcirc)$.

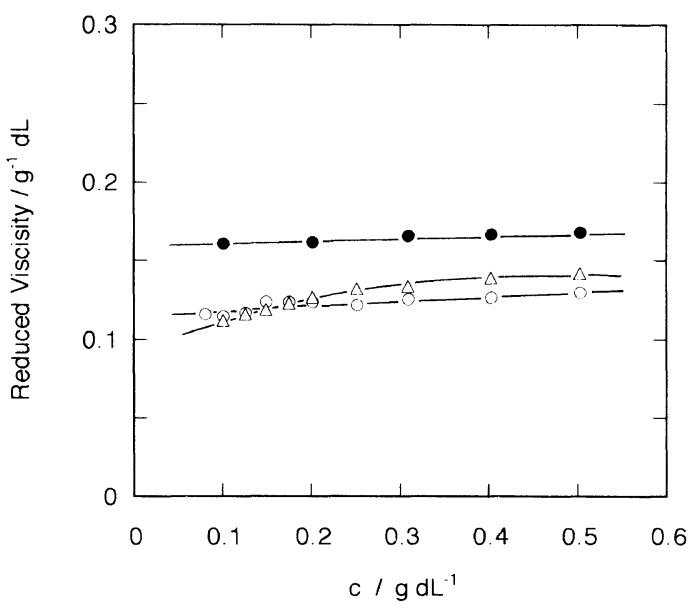

Figure 7. Solution viscosity behavior of $\mathrm{LP} 9 \mathrm{BuCl}$ in toluene with TBHPC at $25^{\circ} \mathrm{C}$ : TBHPC concentration at $5.0 \mathrm{mmol} \mathrm{dm}^{-3}(\mathrm{O})$, and $10 \mathrm{mmol} \mathrm{dm}{ }^{-3}(\triangle)$; LCM9PS without TBHPC $(\bullet)$.

mer concentration range examined. With an increasing concentration of TOMAC, the reduced viscosity of $\mathrm{LA} 7.5 \mathrm{OcCl}$ increased and approached a value of its parent chloromethylated polystyrene, LCM7.5PS, indicating an expansion of the ionomer chain. Similarly, $\mathrm{LP9BuCl}$ was solubilized in THF by the addition of TBHPC and the reduced viscosity of $\mathrm{LP} 9 \mathrm{BuCl}$ was increased with an increasing concentration of TBHPC (Figure 6).

The effect of TBHPC on the viscosity of $\mathrm{LP} 9 \mathrm{BuCl}$ in toluene is shown in Figure 7. Although $\mathrm{LP} 9 \mathrm{BuCl}$ was solubilized by TBHPC in toluene, a significant increase in the reduced viscosity was not observed in the concentration range of $5-10 \mathrm{mmol} \mathrm{dm}^{-3}$ of TBHPC.

The dissolution and the increase of the reduced viscosity indicate the disappearance of inter-ionomer ionic aggregates and intra-ionomer ionic aggregates, respectively. The most probable explanation is the replacement of these aggregates by the aggregates between ionomer ionic groups and the added lowmolecular onium salts. The elimination of the interionomer aggregation would induce the salting-in effect and the decrease in the intra-ionomer aggregation would increase the reduced viscosity.

On the other hand, an addition of low-molecular 


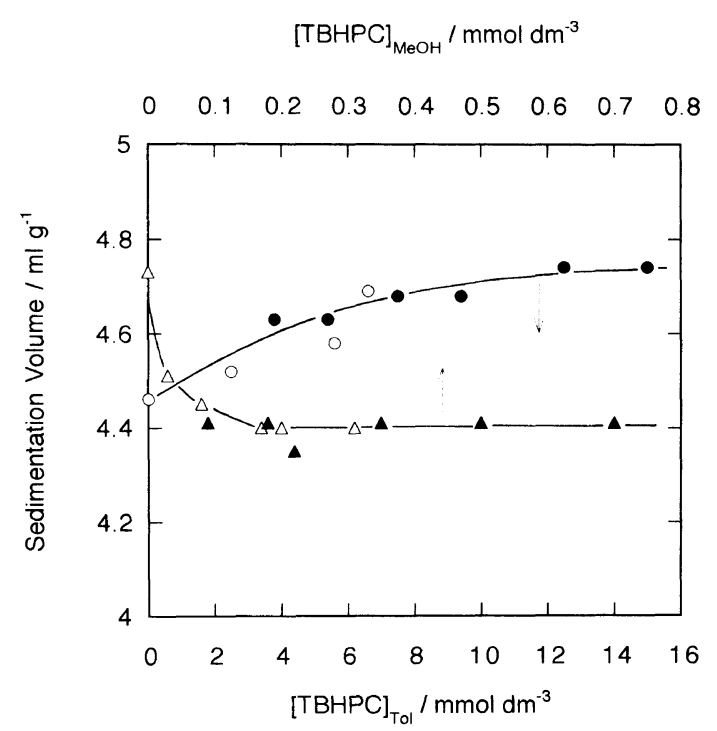

Figure 8. Swelling of $\mathrm{A} 12 \mathrm{BuCl}$ in toluene or methanol with an addition of TBHPC at $25^{\circ} \mathrm{C}$ : in toluene with an increase of TBHPC concentration $(\bigcirc)$ and with a decrease of TBHPC concentration (O); in methanol with an increase of TBHPC concentration $(\triangle)$ and with a decrease of TBHPC concentration $(\boldsymbol{A})$

onium salts into a chloroform solution hardly affected the solution viscosity behavior of any ionomer. The behavior was almost same as that of the parent chloromethylated polystyrene. This supports our previous conclusion that there is no aggregates in chloroform.

Swelling of crosslinked ionomers, $\mathrm{AxOcCl}$, is poor in toluene particularly for the ionomers with large ion contents. This has been explained by the formation of some additional physical crosslinking due to interchain ionic aggregates. Figure 8 shows the influence of TBHPC on the swelling of $\mathrm{A} 12 \mathrm{BuCl}$ in toluene or methanol. In toluene, the degree of swelling was gradually increased with an increasing concentration of the low-molecular phosphonium salts in the supernatant layer. The swelling in methanol presented a contrast to the behavior in toluene. The addition of TBHPC sharply decreased the swelling of $\mathrm{A} 12 \mathrm{BuCl}$ in methanol. In the presence of TBHPC, the swelling ratio became higher in toluene rather than in methanol. The results correspond well to the solubility and viscosity behavior of linear ionomers.
They support the suggested interchain ionic aggregation among ionomer-bound onium salts in nonpolar solvents and the inhibition of the physical interchain crosslinkings in the presence of low-molecular quaternary salts due to the formation of mixed aggregates of ionomer-bound ionic groups and soluble onium salts. The swelling in methanol may be suppressed by the penetration of soluble onium salts due to an increase in hydrophobic character within the resin as well as a decrease in the osmotic pressure.

Acknowledgments. This research was partially supported by Grant-in-Aid for Scientific Research (No. 03650688) from the Ministry of Education, Science, and Culture of Japan.

\section{REFERENCES}

1. S. L. Regen, Angew. Chem., 91, 464 (1979).

2. P. Hodge and D. C. Sherrington, Ed., "Polymer-supported Reactions in Organic Synthesis," Wiley, New York, N.Y., 1980.

3. F. Montanari, D. Landini, and F. Rolla, Top. Curr. Chem., 101, 147 (1982).

4. N. K. Mathur, C. K. Narang, and R. E. Williams, "Polymers as Aids in Organic Chemistry, Academic Press," New York, N.Y., 1980

5. W. T. Ford and M. Tomoi, Adv. Polym. Sci., 55, 49 (1984).

6. N. Ohtani, J. Synthetic Org. Chem., Jpn., 43, 313 (1985).

7. N. Ohtani, C. A. Wilkie, A. Nigam, and S. L. Regen, Macromolecules, 14, 516 (1981).

8. N. Ohtani and S. L. Regen, Macromolecules, 14, 1594 (1981).

9. N. Ohtani, K. Chida, H. Serita, T. Matsunaga, and C. Kimura Bull. Chem. Soc. Jpn., 61, 4371 (1988).

10. N. Ohtani, M. Nakaya, K. Shirahata, and T. Yamashita, J. Polym. Sci., Polym. Chem. Ed., 32, 2677 (1994).

11. N. Ohtani, Y. Inoue, H. Mizuoka, and K. Itoh, J. Polym. Sci., Polym. Chem. Ed., 32, 2589 (1994).

12. M. Hara, J.-L. Wu, and A. H. Lee, Macromolecules, 21, 2214 (1988).

13. H. Morawetz, "Macromolecules in Solution," 2nd ed, Wiley, New York, N.Y., 1975, Chapter 7.

14. N. Ise, Angew. Chem., 25, 323 (1986).

15. N. Ohtani, Y. Inoue, Y. Kaneko, and S. Okumura, J. Polym. Sci., Polym. Chem. Ed., 33, 2449 (1995).

16. V. Gutmann, "The Donor-Acceptor Approach to Molecular Interactions," Plenum Press, New York, N.Y., 1978.

17. P. Outer, C. I. Carr, and B. H. Zimm, J. Chem. Phys., 18, 830 (1950). 